\title{
DINÁMICA INSTITUCIONAL DE LA REPRESENTACIÓN. INDIVIDUALISMO Y PARTIDOS EN LA CÁMARA DE LOS DIPUTADOS*
}

\author{
Carlos Alberto Marques Novaes**
}

Este texto es producto de una investigación sobre la dinámica parlamentaria en la Cámara de los Diputados, y que forma parte del proyecto de investigación que el Grupo de Estudios Políticos del Centro Brasileiro de Análise e Planejamento (CEBRAP) desarrolla bajo la dirección de los profesores Guillermo O'Donnell y Vilmar E. Faria, a los cuales el autor debe más de lo que se podría expresar en una cita de este tipo. Concebido y coordinado por la Dra. Argelina Cheibub Figueiredo, el proyecto "Terra Incógnita: O Congreso Brasileiro" está financiado por la Fundación Melon. La versión inicial de este trabajo recibió valiosos comentarios de Vilmar E. Faria, Argelina Figueiredo, Rodrigo Naves, Álvaro Comin, Eugênio Diniz, Fernando Limogni, Simone Diniz y Yumi Gonçalvez. Por otra parte, si no hay indicación específica, las declaraciones que aparecen en el texto fueron realizadas ante el autor.

\section{INTRODUCCIÓN}

Aunque se disponga de una ponderable literatura sobre los sistemas de partidos y electoral brasileños, e incluso se cuente con algunos estudios que aluden a las implicaciones de dicha normativa sobre la dinámica de la Cámara de los Diputados, existe un significativo silencio sobre la actuación intra muros de los representantes de la ciudadanía brasileña. Este ensayo tiene el propósito de arrojar alguna luz sobre la comprensión de estos aspectos de nuestra vida institucional, asumiendo como punto de partida la premisa que vincula democracia consolidada con el funcionamiento de partidos igualmente consolidados, ya que ésta es la manera más democrática y más eficaz para representar colectivamente intereses políticos en el plano institucional.

Dicho esto, cara a nuestro propósito conviene destacar cuatro inadecuaciones razonablemente conocidas de nuestro sistema partidario, siempre pensando según las exigencias de una democracia deseada':

Traducción del portugués: Ariel Jerez Novara

*) La edición del artículo ha tenido que prescindir, por su excesiva extensión, de dos apartados que versaban sobre la específica dinámica de los Grupos Parlamentarios y la Junta de Portavoces de la Cámara de Diputados brasileña. Los apartados publicados, que son una muestra de la valiosa información y las acertadas reflexiones de una investigación desarrollada a partir de un minucioso y rico trabajo de campo, sirven para introducirnos en la vida del poder legislativo federal brasileño al tiempo que conservan la intencionalidad crítica del autor. Por otra parte, es necesario apuntar que el estudio se realizó durante la anterior legislatura, que ha sido renovada en 1994. (Nota de los editores).

**) Politólogo, investigador del Cebrap. Realiza estudios de posgrado en el departamento de Ciencia Política de la Universidad Federal de Minas Gerais.

(1) Ver Mainwaring, S., "Políticos, partidos e sistemas electorais", Novos Estudos, $\mathrm{n}^{0}$ 29, 1991. CEBRAP, Sao Paulo.
1. Nuestra legislación no distingue entre el derecho para formar un partido y el derecho del partido para disponer de representación en la Cámara.

2. No existe ninguna regla respecto al cambio de partido por parte del parlamentario, estándole permitido hacer todos los cambios que considere oportuno.

3. No existe ningún tipo de compromiso, sea tácito o explícito, consagrado por la costumbre o por la norma jurídica, que vincule al titular del mandato legislativo con el programa del partido que lo eligió.

4. Todo titular de un mandato popular es candidato nato a la próxima elección, teniendo el derecho irrevocable (salvo, naturalmente, en el caso de pérdida de los derechos políticos) de figurar en la lista del partido mediante el cual se le eligió, incluso aunque haya sido expulsado de éste.

La legislación brasileña inhibe toda posibilidad de realización del espíritu agregador que subyace a la idea de partido político, y hace posible que todo arribista, lo suficientemente ambicioso como para buscar en un círculo no muy amplio de amistades las firmas necesarias, disfrute de la posibilidad de disputar una escaño en la Cámara Federal, evitando así el esfuerzo de vencer las resistencias propias de la vida interna de un partido de masas. Por muy viciada que se pueda presentar, esta disputa interna funciona como un filtro indispensable para contener los aventureros opuestos al compromiso, cuya acción en el escenario democrático encierra un daño potencial mayor que el que pueda existir en el propio entramado de las organizaciones complejas sujetas a competición (considerando que estas burocracias generan problemas de otro orden).

Al estimular la proliferación de partidos originados en ambiciones personales no domesticadas, el legislador brasileño, que no es otro sino ese conjunto de representantes cuyas prácticas trataremos a lo largo del texto, engendra una permisiva libertad de acción que incide dentro de la Cámara en un sentido pernicioso para la consolidación democrática, en la medida en que favorece una práctica atomizada de diligencia y proposición legislativa, y una altísima concentración decisoria. Por un lado, los derechos individuales ganan en este espacio una vigencia tan disparatada (al mismo tiempo que existe una adaptación tan extravagante del principio de que a cada ciudadano le corresponde un voto, principio totalmente ignorado cuando se trata de 
un ciudadano común ${ }^{2}$ ), que termina por desaparecer la demarcación entre los intereses del individuo (que es diputado) y los propósitos del diputado (que es representante de los individuos). Así pues, se tiene un escenario en el que se abren paso "miembros" de aproximadamente 18 partidos diferentes, cuyo funcionamiento, en muchos casos, muy remotamente podría ser clasificado como propiamente partidista ${ }^{3}$. Por otro lado, en aparente contradicción con este individualismo, las decisiones finales se toman en rituales que reúnen una parcela reducida de estos individuos que, sin embargo, cuentan con el consentimiento del conjunto.

Las influencias externas que condicionan la dinámica interna de la representación serán analizadas en este texto sólo en el momento en que aporten elementos para la comprensión orgánica de la Cámara, y sin perder de vista el enfoque institucionalista de la investigación, ya que la compleja trama propiamente institucional que nos ocupa rebasa aquellos condicionantes y termina por encerrar lógicas divergentes: si por un lado existe un potencial atomizante procedente de la legislación electoral y partidaria, por otro nos encontramos un ánimo agregador propio de las organizaciones complejas de representación de intereses, cuya eficiencia exige coordinación interna. Es del enfrentamiento y de las combinaciones de estas dos dinámicas de donde surge el interés analítico de la investigación que dio origen a este texto, pues se trata de explorar en el hibridismo institucional de la Cámara baja, que marcada por la concatenación imperfecta de las estrategias sedimentadas a lo largo de los años autoritarios con los impulsos de renovación recibidos en la redemocratización y, principalmente, con los cambios puestos en marcha durante el proceso constituyente, padece al mismo tiempo autoridad por exceso y por defecto. En exceso, porque el poder concentrado por los "notables" (diputados que, con o sin cargos reglamentarios, por su trayectoria personal dentro de la Casa tienen el poder para organizar y articular al interior de sus grupos acuerdos para coordinarlos con otros partidos) actúa en detrimento de una formación colectiva de las preferencias; por defecto, porque la centralización de hecho existente se alimenta más de la fragmentación de lo que la combate. Veamos esta cuestión por partes.

\section{A. La herencia del periodo militar}

La Cámara sufre todavía hoy las medidas adoptadas por los gobiernos militares, tanto en el ámbito externo como interno del parlamento. En el primer caso, la reforma tributaria del régimen militar, forjada en el periodo 1965-67 y mantenida hasta 1988, concentró en el ámbito federal y, más precisamente, en manos del Poder Ejecutivo, la mayor parte de los ingresos tributarios del país. La adopción de esa política tuvo un gran impacto sobre el tipo de expectativas y presiones a las que quedarían sujetos los diputados. Dependientes de la Unión, ya sea por transferencias gubernamentales ya sea por las inversiones directas, los estados y municipios se vieron frente a la necesidad de multipli- car sus vías de acceso al gobierno federal, que pasó a manejar más del $60 \%$ de los tributos y casi el $70 \%$ de los ingresos corrientes federales ${ }^{4}$. Siendo los únicos cargos en disputa electoral durante la dictadura, los mandatos legislativos proporcionales fueron transformados, en la esfera federal, en canales para la negociación entre las instancias subfederales y los responsables de los recursos financieros estatales. Los diputados serían todavía más importantes en la relación de los estados y los municipios con las autoridades ejecutivas federales, ya que podían decidir, por ejemplo, la cantidad y el destino de las transferencias no tributarias (negociadas), que pasaron a lo largo del periodo $1965-1987$, del $0,16 \%$ al $2 \%$ del PIB $^{5}$.

Dado que la definición de las políticas de inversión se realizaba por medio del Plan Nacional de Desarrollo (PND), que era impuesto por el poder ejecutivo sin contar con la participación de la Cámara de los Diputados, —centralización que reflejaba el ideario autoritario de un proyecto nacional, en la medida en que percibía a estados y municipios como competidores indeseables - la vida interna del legislativo fue siendo progresivamente alterada: los diputados empezaron a ejercer sus mandatos más fuera que dentro de la Cámara, pues no sólo los recursos estaban concentrados en el ámbito del ejecutivo federal sino que tampoco dependían de la acción del legislativo como poder. Este reacomodo político tuvo como resultado, cara a lo que aquí nos interesa, aquello que es descrito por uno de los diputados brasileños más informados en los siguientes términos:

“ el universo de trabajo del parlamentario eficaz ya no era el pleno de la Cámara, sino la antesala de los ministros del área económica, los que tenían el dinero y podían invertir en las regiones de procedencia de los diputados (...) El Congreso aprobaba los medios para que el Ejecutivo alcanzase objetivos ya definidos" ${ }^{\circ}$.

Los cambios introducidos en la dinámica interna de la Cámara constituyen lo que analítica y retrospectivamente puede definirse como proceso de atomización de la práctica parlamentaria, es decir, un proceso en el cual la acción individual es progresivamente estimulada en la medida en que ésta es más eficaz que una eventual acción colectiva coordinada. Al lado de la fuerza gravitacional del Ejecutivo federal, la rotatividad introducida en los puestos de coordinación legislativa contribuyó para lo que terminó siendo una falta de tradición, de continuidad, en el proceso legislativo. En la medida en que se prohibió la reelección en los cargos de la Mesa de la Cámara y para los puestos de coordinación de las comisiones técnicas, la bruta concentración de poderes reservada a los titulares de estos cargos pasó a ser empleada por agentes que tenían un tiempo limitado: se trataba de extraer el máximo beneficio en un corto periodo de tiempo?. Un Legislativo desprovisto de funciones ordenadoras generales, capaces de producir efectos de largo plazo - definidos como órbita exclusiva de los PND's diseñados por el Ejecutivo- y estimulado a no establecer rutinas parla-
(2) Estableciendo un máximo y un mínimo en el número de representantes de cada estado de la federación, la legislación produce distorsiones en las que un diputado de Roraima, el estado menos poblado de Brasil, representa a nueve mil electores, mientras que en Sao Paulo un diputado representa a más de 300 mil. Para un tratamiento amplio de éste y otros temas conexos ver Marconi Nicolau, J., Sistema Eleitoral e Reforma Política, Ed.Foglio, Rio de Janeiro, 1993.

(3) Dada la volatilidad partidista es difícil precisar su número, en la medida en que hay partidos que con un diputado pueden perder con él su representación en la Cámara, pudiendo también recuperarla en el caso de que éste vuelva o se inscriba en el partido algún diputado cuyo interés ha sido contrariado en el partido por el que fue elegido.
(4) Afonso, J. R.R., "Aspectos Conceituais das Relaçoes Financeiras Intergovernamentais", Estudos Económicos, $\mathrm{n}^{\mathrm{0}}$ 1. Vol.22, 1992, Sao Paulo.

(5) Afonso, José. R.R., "Evoluçao das relaçoes Intergovernamentais no Brasil entre 1968/1988: transferencia e endividamento", Tesis de Maestrado, Universidade Federal do Rio de Janeiro, Instituto de Economía Industrial, Rio de Janeiro, 1989.

(6) Diputado Nelson Jobbim (PMDB-RS), en declaraciones en CEBRAP en junio de 1993.

(7) El diputado Miro Teixeira (PDT-RJ) supone que "no interesaba (a los militares) consolidar liderazgos. Esta rotatividad (en la Mesa y en las comisiones) es la misma que el General Geisel introdujo en las FF.AA.(...). Geisel no quería correr el riesgo de ver surgir dentro de las FF.AA. liderazgos que afectasen a la dinastía sucesoria (...). Se ponía en marcha el mismo sistema”. 
mentarias que superasen, en algunos casos, más de una sesión legislativa (un año), difícilmente podría controlar la fragmentación potencialmente contenida en el principio de que el voto es el vínculo sagrado entre representante y representado.

\section{B. Relaciones con el Ejecutivo}

La meta número uno del diputado es la reelección ${ }^{8}$. Como las posibilidades de satisfacer a los electores y de responder a los compromisos engendrados en la compleja cadena de vínculos electorales que compone el peculiar federalismo brasileño -peculiar porque en Brasil los municipios tienen una importancia superior a la encontrada en otras federaciones- sólo están al alcance de los parlamentarios dispuestos a gravitar en torno al Ejecutivo, se produce lo que un experimentado diputado describe en términos muy realistas:

"el diputado es, en general, un procurador de partes, que no tiene la menor participación en el Pleno, que sólo va a los ministerios. Oficialista por vocación y por necesidad, él intenta conseguir a lo largo de los cuatro años de su mandato recursos para sus municipios, preocupado por su reelección" 10 .

Este estado de cosas echó raíces profundas y constituye todavía hoy la pauta del vínculo existente entre los diputados y sus redes de apoyo. Ni siquiera el Partido dos Trabalhadores-PT, agrupación de izquierda que en muchos aspectos es una novedad en el escenario partidista brasileño, escapa a las determinaciones estructurales de este proceso:

"La presión sobre los parlamentarios para conseguir recursos presupuestarios existe, nosotros tenemos allá en Londrina el ayuntamiento del PT. Nuestro alcalde hace reuniones con varios diputados, conmigo, para pedir empeño y conseguir recursos de la Unión para el municipio. Eso existe. El parlamentario que no consigue articular aquí algo, él prácticamente no existe, porque del punto de vista de las parroquias, vamos a llamarle así, él no está haciendo nada" ".

A continuación veremos que las modificaciones aprobadas en la Constitución de 1988, finalizado el ciclo militar, introducen lógicas parciales nuevas que compiten con la tradición heredada de la lógica anterior. Un ejemplo es la reducción de la capacidad tributaria de la Unión, que limitó las posibilidades materiales del Ejecutivo para ejercer esa atracción viciosa sobre los parlamentarios. Así, al lado de las presiones de procedencia subfederal (una necesidad inercial legada por la tradición centralizadora anterior), ahora los diputados son asediados por ministros y otras autoridades del Ejecutivo dentro del propio parlamento, que, en días de votaciones decisivas, despachan en los gabinetes de los cargos de Grupo Parlamentario, hecho denunciado en el Pleno por un diputado de la oposición y descrito en los siguientes términos:

(8) Aunque algunos diputados constituyentes no se hayan presentado para competir en las elecciones siguientes, es erróneo deducir de ello que su objetivo, a lo largo del mandato, no haya sido la reelección. Por razones que no estoy en condiciones de desarrollar por falta de informaciones precisas, la volatilidad de los lazos que permiten una candidatura exitosa es muy alta y, claro, esta cuestión entra en los cálculos de un postulante realista, lo que lleva a que muchos de ellos desistan al considerar los costos materiales de la campaña.

(9) Los municipios tienen autonomía administrativa y tributaria, y no son una instancia de poder subordinada a la coordinación estadual. Su fuerza palítica se expresa en el plano nacional por medio del Frente Nacional de Prefeitos, que representa la continuidad de una tradición que se remonta al periodo imperial brasileño.

(10) Diputado Israel Pinheiro Filho (PMDB-MG)

(11) Diputado Paulo Bernardo (PT-PR)
"Hemos visto ya (...) al Presidente de la Caixa Económica Federal, al Presidente del Banco do Brasil, a algunos ministros, venir al Pleno del Congreso Nacional para contar los votos, para conversar con los diputados, para conceder créditos justo ese día para el Diputado" ${ }^{12}$.

\section{PERFIL GENERAL DE LA REPRESENTACIÓN Y DE LOS PARTIDOS}

Como hemos mencionado, la baja intensidad de la vinculaciones propiamente partidistas, que darían sentido colectivo a los mandatos individuales, refleja una legislación muy concreta para la organización de los partidos. Además, es necesario tener en cuenta que cada partido puede hacer en los diferentes estados las coaliciones electorales que bien entienda, sin obedecer a ningún criterio nacional. La excepción, entre los seis partidos que poseen los mayores grupos parlamentarios, es el PT, el único que aprueba una política de alianzas de carácter nacional, aunque viene enfrentando problemas para las elecciones de $1994^{13}$ En los demás partidos lo usual es dejar que cada estado defina su propia estrategia de campaña. Paralelamente, tampoco contamos con una exigencia legal que vincule las coaliciones electorales a las posteriores relaciones parlamentarias entre los diferentes Grupos.

No obstante, es frecuente que los alineamientos que se observan durante las votaciones nominales en el Pleno, tengan bastante correspondencia con las alianzas electorales realizadas. Esta correspondencia no es reflejo de una estructura orgánica de partidos: a nuestro gelatinoso orden partidista, que sufre el problema añadido de una altísima tasa de renovación, subyace un individualismo que prevalece en la actuación cotidiana del parlamentario. Veamos esto con más detalle.

\section{A. Las características de la renovación}

Si hay algún dato que decisivamente interfiere en la dinámica interna de la Cámara de los diputados es la altísima renovación de una legislatura a otra. De la Asamblea Constituyente a la actual legislatura, la renovación ha sido de más del $60 \%$, llegando los diputados de primer mandato (que no habían sido diputados antes de la Constituyente) al 54\%. No es cuestión de abordar aquí las razones que el electorado tiene para actuar de esta manera, aunque cabe señalar que en general esta actuación es valorada como positiva por la opinión pública, que ve en los "políticos" a los responsables de la crisis e imagina la renovación simultáneamente como castigo y promesa de días mejores. No obstante, analizada desde el punto de vista interno, la renovación trae consigo problemas que, en cierta forma, han conspirado contra las esperanzas del elector. La acción parlamentaria, en tanto actividad que requiere el conocimiento de una minuciosa rutina, sufre la incidencia de los apetitos indisciplinados derivados de la renovación, configurándose un cuadro en el que es todavía más complejo lograr cualquier pretensión de actividad coordinadora. Además, la presencia de una mayoría de novatos facilita el trabajo de aquellos que controlan de hecho el

(12) Diputado Chico Vigilante (PT-DF).

(13) La paulatina inserción del partido en las lógicas electorales estatales va poniendo a su direcció delante de los dilemas que una burocracia partidista nacional siempre enfrenta en un país federativo. 
proceso legislativo, beneficiados por un proceso de delegación interna que se apoya en buena parte en la ignorancia funcional de los nuevos diputados. Reproduciendo la naturaleza personalista de la dinámica legislativa, la Cámara no cuenta con ninguna forma de recepción instructiva para los diputados de primer mandato, por lo que existe un total silencio sobre el asunto en sus normas internas, salvo una presentación burocrática promovida por la administración general de la Casa. En esta presentación los funcionarios viajan a los estados de origen de los diputados electos para, en una entrevista individual, explicar cuestiones de la vida en Brasilia, colegio para los hijos, la geografía de la Cámara, los departamentos, etc. Mientras tanto, nada se dice del Reglamento Interno y de la actividad parlamentaria como tal. Muchos entrevistados justifican esta negligencia institucional argumentando que esta tarea formativa corresponde a los partidos políticos.

Obviamente, los partidos tampoco llevan a cabo ninguna actividad para aclimatar colectiva o individualmente a los nuevos diputados. Este "descuido" es producto del modelo partidista y electoral por el cual los diputados fueron previamente candidatados y, posteriormente, enfrentados en la arena electoral. Por tanto, el camino personalista precedente tendrá continuidad en la Cámara: de los diez mayores grupos parlamentarios, sólo el PT se ocupa planificadamente de los diputados de primer mandato ${ }^{14}$; en los otros grupos las prácticas pueden ser resumida, según nuestra investigación, en las palabras de un diputado del PPR: "cuide cada uno de sí, que ya cuidará Dios de todos".

Los efectos negativos de la ausencia de recepción institucional serían menores si para los diputados de primer mandato la Cámara significase de algún modo la culminación de una experiencia legislativa previa, bagaje que no los dejaría en la condición de neófitos frente a la nueva actividad representativa. En este sentido, el cuadro resulta desalentador cuando se observan los siguientes datos: el $76 \%$ de los diputados federales de primer mandato nunca ejercieron el cargo de concejal; el $65 \%$ de ellos nunca fueron diputados estatales y el $54 \%$ no ejercieron ninguno de los cargos. Si observamos esto en el modus vivendi de la Cámara, vemos cómo ese perfil se ajusta a los objetivos de aquellos que pretenden dejar en pocas manos la conducción de los muchos intereses en juego, pues es necesario un experiencia ajena a los novatos para discernir y, después, reivindicar una parcela de poder disponible para la promoción personal, política y material. Como dice un diputado habituado a las reelecciones:

"el diputado llega aquí y pasa tres años en la búsqueda del camino, y cuando lo encuentra ya está derrotado, porque se tiene que encargar de la reelección. Entre encontrar el camino y conseguir la reelección, se queda en el medio. No encuentra ni lo uno ni lo otro ${ }^{15}$ ".

\section{B. Perfil de la actividad parlamentaria individual}

Además de tener a su disposición un grupo de asesores en los despachos individuales, los diputados cuentan con una asesoría general de la Cámara que, en principio, debería servir al proceso legislativo como tal. El uso que los diputados hacen de

(14) La asesoría del Grupo produce una guía que resume didácticamente los principales puntos de Reglamento Interno, con explicaciones adicionales sobre la actividad parlamentaria en general. Además, se promueven reuniones especiales con los diputados de primer mandato

(15) Diputado Israel Pinheiro (PMDB-MG). la Asesoría Legislativa (AL), órgano técnico general que funciona en la Cámara para apoyarlos en sus actividades de representación, ilustra el particularismo de la vida parlamentaria, incluso después de los cambios introducidos en la nueva Constitución. Los servicios prestados por la AL son de cuatro tipos: la "elaboración legislativa", que incluye los proyectos de ley en sí mismos; la "redacción parlamentaria", que consiste en la elaboración de discursos; la "investigación", donde aparecen los trabajos temáticos de mayor duración, y, finalmente, el "servicio a las comisiones", mecanismo puesto en marcha después de la Constituyente, y que ha revalorizado el papel de éstas.

Los siguientes gráficos muestran la participación de cada uno de estos tipos de servicios en el conjunto de los trabajos solicitados, con la excepción de los datos sobre las comisiones, que son aproximados.

ASESORÍA LEGISLATIVA

Tipos de trabajo solicitados

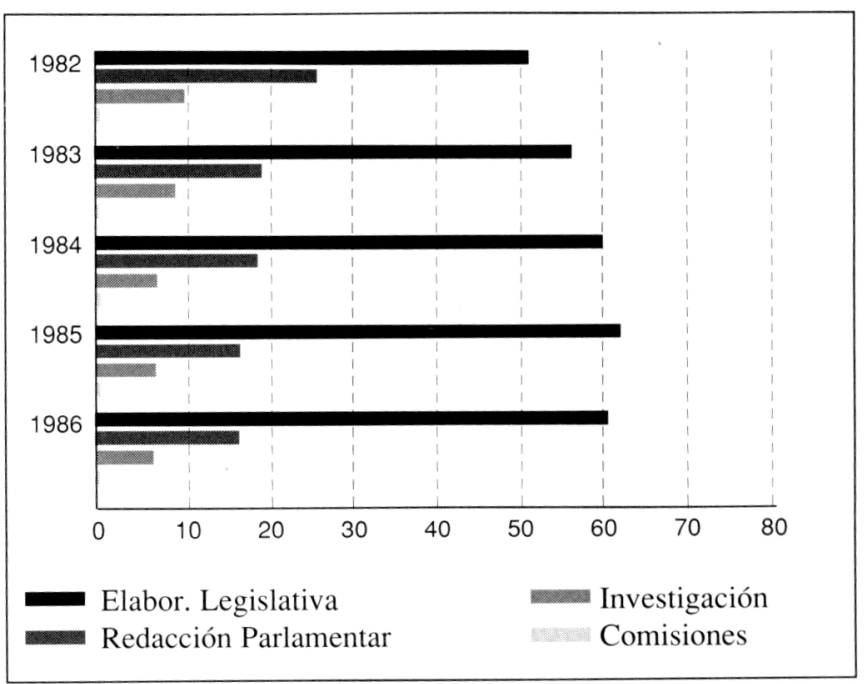

ASESORÍA LEGISLATIVA

Tipos de trabajo solicitados

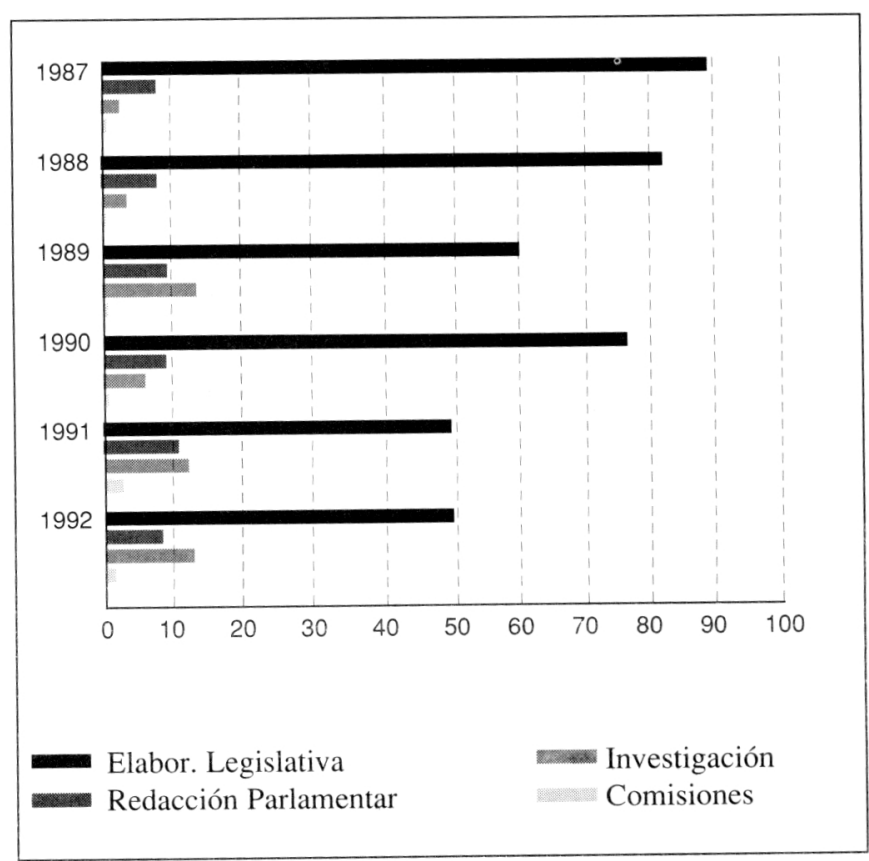


Como se puede observar, la participación de las peticiones de redacción parlamentaria caen bastante si comparamos el periodo preconstituyente (1983-1986) con el bienio en el que se elaboró la constitución (87-88). No podía ser de otra manera, ya que esos dos años no se dedicaron al proselitismo en el Pleno: se trataba de discutir, negociar y reorientar la nueva Carta en las comisiones y subcomisiones temáticas. En los dos últimos años de la legislatura constituyente y en los dos primeros de la legislatura siguiente, la participación de las peticiones de redacción no volvieron a alcanzar los niveles habituales que se remontan a 1878. Empujados por el nuevo contexto, que también afectó la percepción que los asesores tenían de su propio trabajo, los diputados han ido alterando el tipo de demanda que hacían a la AL, prestando más atención a la investigación y a la elaboración legislativa. Sus peticiones también cedieron un pequeño espacio al trabajo de las comisiones, que en el bienio 91-92 tienen una participación de entre el $10 \%$ y el $15 \%$ de las solicitudes en la AL, algo hasta entonces inédito. Si, por un lado, esa alteración en los números puede indicar una evolución en la conducta parlamentaria, por otro, al examinar las mismas peticiones según su procedencia, se observa que el cuadro varió mínimamente:

ASESORIA LEGISLATIVA

Autoría de pedidos de asesoramiento

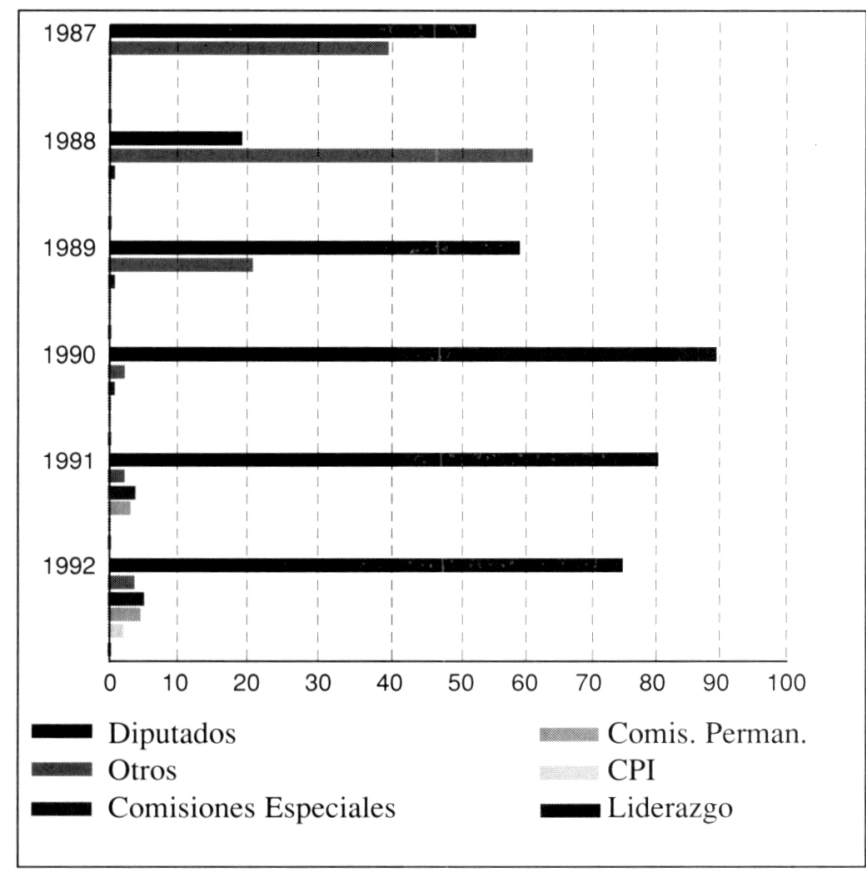

Como se puede observar en este gráfico, las peticiones individuales de los diputados no constituyen menos del $80 \%$ del total de los servicios prestados, salvo para los tres años en los que la Mesa fue un gran solicitante ("otros", en el gráfico), coincidiendo con el bienio constituyente, y del año posterior, en los que se dependió mucho de la AL en la medida en que dirigía el desarrollo de muchos dispositivos constitucionales que requerían (y requieren) una legislación específica. El uso colectivo de la AL, que señalaría una práctica parlamentaria agregada, no llega al $15 \%$ de los servicios prestados por ésta. Aunque se hayan recibido nuevas e importantes prerrogativas, las comisiones han tenido dificultad para hacer valer sus necesidades. Llama la atención la participación irrisoria de las peticiones de los grupos parlamentarios. Si tenemos en cuenta que las peticiones individuales de redacción parlamentaria son, en el $70 \%$ de los casos, peticiones de discurso para los llamados "pequeños expedientes" (periodo de sesión plenaria en el que los diputados pueden hablar cerca de cinco minutos sobre un asunto de libre elección), tendremos un cuadro aproximado al grado de dispersión en que se ejerce un mandato de diputado federal en Brasil. Respecto a este particular es interesante registrar un dato que anticipa aspectos de los que se va a discutir posteriormente: cuando se tiene en cuenta la participación proporcional de los partidos solicitantes, se descubre que los grupos que presentan la práctica colectiva más desarrollada son el PT y el PCdoB, siendo los grandes partidos los que menor uso colectivo hacen de la AL.

$\mathrm{El}$ modo en que la propia AL visualiza sus problemas es ilustrativa de la "estructura de atomización". Según los datos recogidos, en el año 1991 sólo el 23\% de los parlamentarios no había solicitado servicios a AL, en contraste con la concentración de las demandas en algunos nombres. Cuando la AL descubre esta situación se plantea como tarea primordial "realizar un amplio programa de divulgación de la Asesoría junto a los parlamentarios, de forma que se estimule a una mayor número en la solicitudes de ayuda a este órgano" ${ }_{16}$, propuesta contraproducente, en la medida en que desea estimular el uso individual de los servicios, agravando un modelo nefasto.

Muchos parlamentarios entrevistados entienden que el problema reside en la forma en que la AL está organizada. Para solventar esta situación, tendría que dejar de ser un órgano con funcionamiento autónomo, que no posibilite el acceso individual de los diputados, desarrollando sus trabajos directamente en las comisiones y en los grupos parlamentarios. La reestructuración implicaría distribuir el actual cuerpo único de asesores en las comisiones, según cualificaciones y requerimientos técnicos. Pero estas transformaciones en la AL difícilmente alcanzarán su objetivo de alterar la dinámica parlamentaria, si la concepción y la práctica de un mandato popular proporcional no son obligadas a atenuar su corte individualista. Ese individualismo se explica no por el afán del diputado en participar del proceso legislativo en sí mismo, sino por la necesidad de "mostrar buena disposición". En general, los proyectos son presentados no para cumplir toda su tramitación parlamentaria, sino para que el autor, una vez en posesión de las pruebas documentales expedidas por la Cámara, pueda hacer propaganda junto a su clientela de los "esfuerzos" realizados por sacar adelante esta o aquella cuestión. Una vez registrados los "avulsos" "17, el diputado va a gastar su franquicia de correo enviándoselos a sus bases y, en la inmensa mayoría de los casos, se desentiende de la suerte del proyecto, que ni siquiera llegará a su deliberación final. Esta es parte de la explicación del hecho de que habiéndose presentado 6.601 proyectos de ley en el periodo $89-91$, los diputados hayan aprobado 43 de ellos.

Además de tener que enfrentar estos obstáculos de carácter general, quién pretenda cambiar el uso de la $A L$ se tendrá que enfrentar a la oposición de sus funcionarios, que, como toda burocracia, ven cualquier reestructuración que signifique desorden en el monolito organizativo como una amenaza a sus intereses. Esta "disputa” está en marcha y señala un problema todavía

(16) "Perfil da demanda de trabalhos Assesoría Legislativa", Directorla Legislativa, 12 de diciembre de 1991, mimeo.

17) Avulso es el término técnico con que se designa el impreso que sirve para publicitar los proyec tos en tramitación. 
mayor: las dificultades encontradas por los reformadores para controlar la burocracia general de la Cámara que, por su parte, son producto de los dilemas surgidos de la renovación y de la fragmentación. Mucho más estable y con intereses susceptibles de ser coordinados con mayor facilidad, la burocracia general saca provecho tanto de la renovación como de la fragmentación de los diputados para hacer valer sus poderes. En el primer caso, como ya mencionamos, se encarga de la recepción de los diputados de primer mandato, estableciendo con ellos lazos que pasan como favores, tales como adelantos de sueldo, préstamos para su instalación en Brasilia, la obtención de un buen gabinete, la asignación de funcionarios experimentados en la trama legislativa y una serie de otras facilidades que son recibidas con gratitud por el novato aislado. En el segundo caso, el de la fragmentación, la burocracia no sólo la refuerza cuando teje su red en la ausencia de una partidista, sino que se beneficia de ella, como es el caso de la última elección de la Mesa de la Cámara.

Al igual que en los parlamentos de las grandes democracias, también entre nosotros la Mesa y su Presidente tiene grandes poderes sobre el día a día parlamentario. En la disputa por su control en el bienio 93-94 había dos campos nítidos. Entre sus diferencias se destacaba la consideración sobre el lugar que la burocracia de la Casa debería ocupar, de los poderes que debería disponer. Relatos de varios entrevistados coinciden en lo siguiente: la burocracia se movilizó para favorecer en esta disputa al bloque pro statu quo. Buscó individualmente a los diputados y salió victoriosa:

"hay una nomenclatura atrevida, osada y que contro-

la la Cámara de una manera que interfiere, por ejemplo, en el proceso de elección de la Mesa, buscando a los diputados, yendo a sus despachos. ¿Quién conoce a ese diputado de Bahía?': vayan allá, busquen alguien con base en Bahia, tres alcaldes [por ejemplo], hagan un puente para llegar al diputado. (...) El Presidente Inocêncio de Oliveira fue electo en una elección en la que tenías los Directores de los Departamentos de la Casa dentro del Pleno. Es una cosa infernal y peligrosísima lo que hoy está ocurriendo dentro de la Cámara" ${ }^{18}$.

Naturalmente no se trata de imaginar que los diputados, incluidos los de primer mandato, hayan sido víctimas de una burocracia interesada. Ellos mismos son muy celosos de su "autonomía”, duramente conquistada en el encarnizado individualismo del proceso electoral. Ese individualismo es acentuado por las direcciones partidistas, que buscan candidatos con "gancho electoral" independientemente de los lazos del pretendiente con el partido o de sus convicciones políticas. Como plantea uno de los diputados más preocupados con las inadecuaciones de nuestra legislación electoral,

"el partido depende de sus candidatos y no los candidatos del partido. La elección del candidato que pueda hacer 30 o 40 mil votos se vincula no a la bandera del partido sino a la capacidad individual del sujeto para producir votos" 19 .

Los daños causados por esa búsqueda electoralista de los "ganchos" son agravadas por la permisividad existente para formar coaliciones.

(18) Diputado Miro Teixeira (PDT-RJ)

(19) Diputado Nelson Jobim (PMDB-RS)

\section{Coaliciones electorales y alineaciones intra muros ${ }^{20}$}

Según como se definen y sitúan los propios agentes, la izquierda sería el conjunto PT, PSB, PCdoB, y PPS; el centroizquierda recoge al PDT y al PSDB; la derecha aglutina al conjunto formado por PFL, PPR (PDS+PDC), PRN, PTB, PL, PP (PTR+PST), PSD y PSC y el centro-derecha al PMDB. Considerando las coaliciones que estos partidos realizaron en las elecciones de 1990, que definió la actual legislatura en la Cámara Federal, se observa que en ningún estado hubo coaliciones que reuniesen partidos presentes en los polos opuestos de esta clasificación. Todos los diputados electos en coalición están, según los datos estudiados, o a la izquierda o a la derecha del espectro electoral. De esta manera, llamaremos coaliciones mixtas a aquellas que incluyan partidos de lugares diferentes en el espectro ideológico, pero en un juego de combinaciones que excluye los polos. Las coaliciones de derecha reúnen, además del partido en cuestión, sólo partidos de derecha; las de izquierda igualmente sólo consideran partidos de izquierda; al igual que las centro-izquierda y las de centro-derecha.

El PFL, el PDS, el PRN y el PTB, por ejemplo, que establecieron sólo dos tipos de alianzas exitosas, tuvieron el $82 \%$ de sus diputados electos en alianzas de derecha, aunque tuvieron éxito también en algunas coaliciones mixtas, que en ciertas regiones incluyeron al PMDB. El PMDB tuvo el 59\% de sus 101 diputados electos en alianzas. De estos, el 58\% llegaron a la Cámara después de campañas compartidas con la derecha. El partido hizo dos tipos de alianzas, eligiendo, en tres regiones, diputados en coaliciones mixtas, en esos casos junto a los de centroizquierda.

El PSDB eligió el $62 \%$ de sus 42 diputados en cuatro tipos de alianzas: en algunos estados se unió a partidos de derecha (15\%), en otros sólo con partidos de izquierda (15\%), habiendo logrado éxitos también con los de centro-izquierda (con el PDT, el 23\%), obteniendo el grueso de sus diputados con coaliciones mixtas $(46 \%)$. Por tanto no ha de sorprendernos que en este partido las cuestiones estatales y regionales alcancen, como se verá, una relevancia especial. El PDT eligió sus diputados mediante tres tipos de alianzas: las de derecha ( $7 \%$ del grupo), las de izquierda $(40 \%)$ y las mixtas $(28 \%)$. El PT sólo hizo dos tipos de alianzas, de izquierda y mixta, eligiendo el $75 \%$ de sus 36 diputados en el primer modelo y $11 \%$ en el segundo. Finalmente, para completar este cuadro, excesivamente esquemático, es útil decir que cerca del $80 \%$ de los diputados electos en 1990 lo han logrado con su partido formando alguna coalición.

Hay un cuadro ideológicamente consistente de concatenación de intereses, con la excepción del PSDB y del PMDB, cuyas coaliciones son más heterogéneas. En el caso del PSDB, se trata de un partido de "opinión pública" con escasa inserción entre los sectores organizados, ya sea en el mundo del trabajo, ya sea en el mundo del capital. Originado en una disidencia del

(20) Para lo que sigue es útil informar que: Partido do Movimiento Democr-tico Brasileiro-PMDB (101 diputados), Partido del Frente Liberal-PFL (88), Partido Democrático Social-PDS (58), Partido Democrático Trabalhista-PDT (41), Partido da Social Democracia Brasileira-PSDB (38), Partido Popular (37), Partido dos Trabalhadores-PT (37), Partido Trabalhista Brasileiro-PTB (26), Partido da Reconstruçao Nacional-PRN (16), Partido Liberal-PL (14), Partido Socialista Brasileiro-PSB (9), Partido Social Democrático-PSD (6), Partido Comunista do Brasil-PCdoB (7). Partido Social Crisitiono-PSC (4) Partido Demó crático-PSD (6), Pando Cont a do Trathic Reforide do Trabalhista Reformador-PTR (1), Partido Social Trabalhista-PST (1), Partido Verde-PV (1), Partido de la Reedificación del Orden Nacional-PRONA (1) 
PMDB, heredó de éste la heterogeneidad social e ideológica. Sus líderes nacionales, protagonistas de dicha escisión, profesan ideas progresistas próximas al ideario socialdemócrata patrón, que el partido proyecta en la fase nacional de su imagen pública, mientras las estructuras regionales gozan de la libertad necesaria para llegar a los acuerdos electorales oportunos y que, obviamente, revelan las diferencias internas de este partido. En relación al PMDB, se trata del mayor partido brasileño, que asumió rasgos de partido acaparador gracias a que fue el único que pudo funcionar como oposición durante la dictadura militar. De esta forma se entiende que no transfiriese homogeneidad ideológica a los disidentes, ni que la disidencia abierta por el PSDB aumentase su propia homogeneidad social e ideológica: el corte fue vertical.

¿Si este es el diseño de las coaliciones electorales, cómo se conducen los Grupos de estos partidos en la Cámara de los Diputados? Aunque sean un conjunto muy pequeño de las decisiones tomadas en la Cámara, las votaciones nominales permiten una evaluación útil a nuestros propósitos. Veamos el

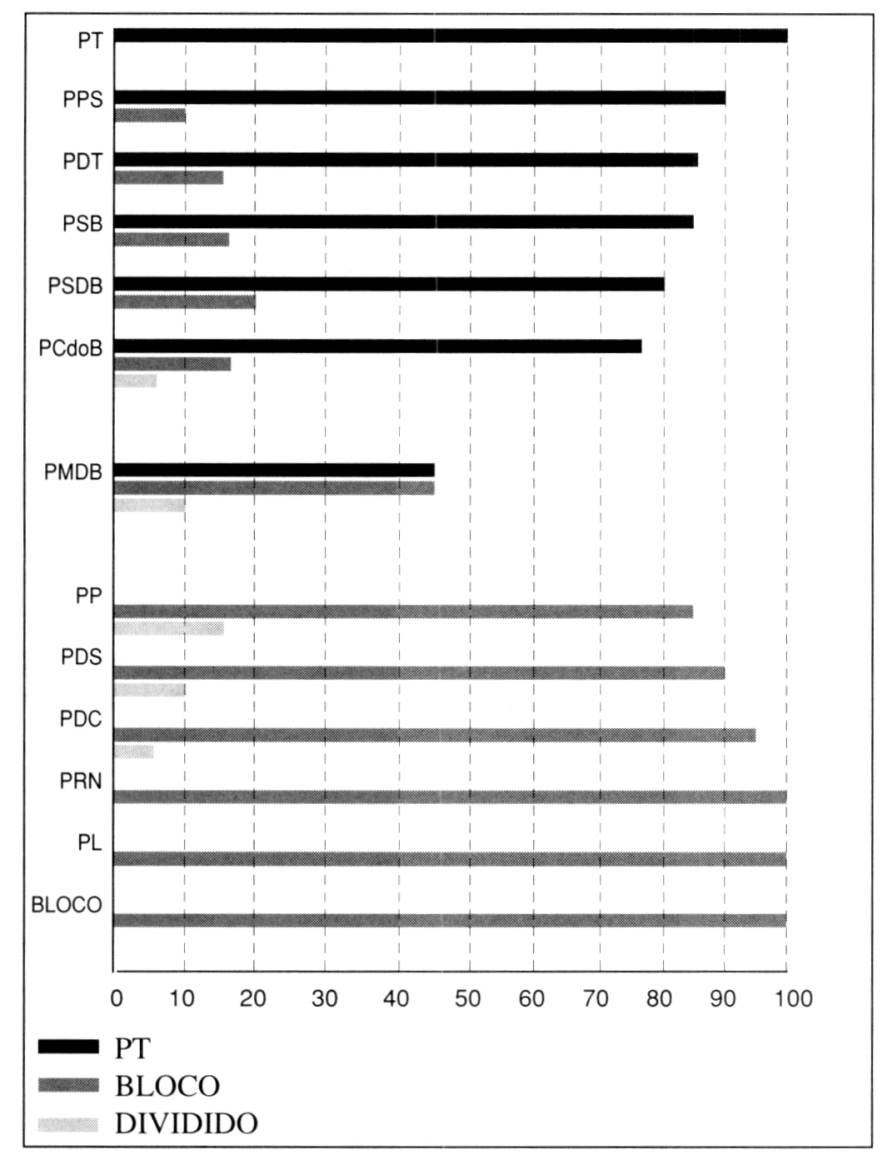

\begin{tabular}{|c|c|c|c|}
\hline \multirow[t]{2}{*}{ PARTIDO } & \multicolumn{3}{|c|}{ ACOMPANHOU (\%) } \\
\hline & $B L O C O$ & $P T$ & DIVIDIU-SE \\
\hline PT & 0,00 & 100,00 & 0,00 \\
\hline PPS & 10,00 & 90,00 & 0,00 \\
\hline PDT & 15,00 & 85,00 & 0,00 \\
\hline PSB & 15,79 & 84,21 & 0,00 \\
\hline PSDB & 20,00 & 80,00 & 0,00 \\
\hline PCdoB & 17,65 & 76,47 & 5,88 \\
\hline PMDB & 45,00 & 45,00 & 10,00 \\
\hline PP & 85,00 & 0,00 & 15,00 \\
\hline PDS & 90,00 & 0,00 & 10,00 \\
\hline PDC & 95,00 & 0,00 & 5,00 \\
\hline PRN & 100,00 & 0,00 & 0,00 \\
\hline $\mathrm{PL}$ & 100,00 & 0,00 & 0,00 \\
\hline BLOCO & 100,00 & 0,00 & 0,00 \\
\hline
\end{tabular}

siguiente gráfico, elaborado a partir de 37 deliberaciones en las que los diputados votaron individualmente en el Pleno en el periodo que va de enero del 91 a enero del 9321. Antes, no obstante, es indispensable informar de lo siguiente: a) el BLOQUE reúne los diputados del PFL, PTB, PSC y PRS; b) el PL y el PT funcionan como parámetros opuestos porque son los dos grupos que más se oponen en las votaciones; c) en la parte superior del gráfico tenemos el conjunto de los votos individuales de los diputados según su alineación con las preferencias mayoritarias del grupo del PT y agrupados por partidos, y en la línea inferior aparece la misma distribución según los votos "acompañen” el conjunto de preferencias mayoritarias del PL.

Como se puede observar, a pesar de que puedan existir disonancias al interior de los grupos, las alineaciones mayoritarias corresponden a los cortes clásicos entre izquierda y derecha, reiterando el diseño ideológico de las coaliciones electorales. Aunque alineándose preferentemente por la izquierda, sin oscilaciones de la magnitud de las del PMDB, los diputados del PSDB acompañaron a los colegas a su derecha en $34 \%$ de los votos que realizaron. Las oscilaciones del PMDB reflejan tanto su composición acaparadora, como su carácter frentista, así como la susceptibilidad del grupo para tener una presencia fluctuante en el Pleno: dependiendo de quién está votando, el grupo cambia efectivamente su "perfil ideológico". La visión de conjunto, mientras tanto, no nos debe llevar a la conclusión de que, al final, la solidez orgánica caracteriza a los partidos políticos en Brasil. En definitiva, la Cámara, que exhibe los nítidos contrastes observados en el gráfico precedente, registra en el periodo analizado (enero del 91 a enero del 93) 174 casos de cambio de partidos entre los diputados ${ }^{22}$. Estos trasvases se produjeron casi siempre en los grupos de la derecha del espectro partidario, y jamás entre grupos situados en los polos opuestos de las coaliciones. Las estructuras partidistas son frágiles, sin embargo las afinidades ideológicas configuran campos estables.

Lejos de configurar una aglutinación orgánica, por tanto, esta correspondencia refleja el gran poder o habilidad de los "notables", que se mueven en un mismo campo ideológico general, aunque en la mayoría de los casos no responden a organizaciones estables y/o consolidadas. En periodos electorales, las direcciones partidistas promueven las coaliciones en el intento de incrementar sus posibilidades de éxito. En Brasil, un sistema proporcional conjugado con lista abierta, en un contexto de total permisividad de alianzas electorales, permite a los miembros de un partido coaligado - que de haber competido en solitario no hubiese alcanzado el mínimo necesario - ser elegidos gracias al refuerzo de votos que la aglutinación produce. Ese cálculo preside la formación de coaliciones, que si bien se orientan regionalmente por las afinidades ideológicas clásicas, paralelamente muestran como esos alineamientos responden a intereses individuales de los miembros de la direcciones partidarias. En la posterior dinámica parlamentaria se observa un proceso elitista semejante: los cargos reglamentarios de los Grupos parlamentarios articulan sin considerar la voluntad de sus compañeros la correspondencia aparecida en el gráfico, más allá de que ésta aparezca con forma de voto individual. De todas formas, no se

(21) Aquí se incluyen votaciones de procedimiento que permiten percibir la falta de coincidencia con los datos de la Tabla 2, que señala para el periodo 89-92 sólo las Leyes Ordinarias sancionadas.

(22) Es oportuno señalar que no se consideran los cambios producidos por la formación del PP y del PPR, ni se incluye el reciente crecimiento del PSD, que adquiere 15 diputados. 
puede afirmar que el alineamiento ideológico se traduzca en organicidad partidista, fidelidad partidista y/o práctica legislativa colegiada. Con este telón de fondo, pasemos a la traducción intra muros de estos problemas.

\section{EL LEGADO DE LA CONSTITUYENTE}

Aunque la Asamblea Nacional Constituyente no haya resuelto el tema de la distorsión en la representación, ni alterado la legislación partidista y electoral en los puntos en los se observan las mencionadas inadecuaciones, sí afectó a la acción legislativa intra muros en tres dimensiones principales: a) por la propia forma de elaboración de la nueva Constitución; b) por el fortalecimiento de los poderes del legislativo; y c) por la redistribución tanto de la capacidad tributaria como de las transferencias intergubernamentales.

\section{A. La práctica de la Constituyente como modelo}

Desde el fin del ciclo militar el país no dispone de una mayoría política que, funcionando orgánicamente en el ámbito federal, articule Ejecutivo y Legislativo y sea capaz de devolver al gobierno, a cualquier gobierno, el manejo eficaz de la capacidad coordinadora del estado en un régimen democrático. El proceso constituyente brasileño fue una expresión cabal de ese vacío político y es frecuente que analistas y políticos digan que la Constitución resulta de un empate entre "progresistas" y "conservadores". Ese empate deriva de la ausencia de una fuerza mayoritaria que, en definitiva, subordine y coordine intereses individuales en el escenario público político brasileño. Tanto es así que fue literalmente imposible preparar cualquier esbozo, anteproyecto o esquema previo de Constitución para presentar a los diputados ${ }^{23}$. Enredados en la rutina de supervivencia resumida en la Introducción, los diputados constituyentes transmitieron en la elaboración de la nueva Constitución las ansiedades que guían su conducta por los laberintos de poder: se trataba, para cada uno de ellos, de garantizar en la letra de la propia Constitución la consagración de los intereses que tenía o canalizaba. Toda tentativa de estructuración previa era rechazada por "autoritaria", habiendo, por supuesto, antiguos aliados de la dictadura entre los más desenvueltos en el uso de ese adjetivo mágico. Para los sectores progresistas, el temor central, no sin fundamento en nuestra tradición legislativa, era que el poder Ejecutivo pudiese influir indebidamente en el proceso.

En la tentativa de ordenar esta desmembrada situación, fueron creadas ocho comisiones temáticas, encargadas de reagrupar las diferentes propuestas. Dentro de estas comisiones se distribuían los diputados, y el resultado fue una "propuesta" de Constitución que el relator agrupó en 501 artículos, los cuales, por su parte, recibieron 977 enmiendas de adecuación y 4.638 enmiendas de mérito. Abierto nuevamente el debate se presentaron a este proyecto preliminar 20.790 enmiendas de los constituyen-

(23) El propio presidente de la República en ese momento, José Sarney (1985-1989), nombró una comisión de intelectuales, políticos y juristas para elaborar un comprometido anteproyecto que, una vez concluido, fue desde el primer momento repudiado por los diputados por considerarlo indebidamente tutelar. El presidente acabó por no mandar el proyecto al Congreso. tes y 122 enmiendas populares ${ }^{24}$, en las cuales todos habían registrado sus pleitos.

En la cúspide de esa atomización fue creada una Comisión de Sistematización, en cuya composición había una mayoría cuasi-fortuita de "progresistas" — esto fue así por razones y mecanismos que no cabe detallar aquí, pero que sólo tuvieron lugar por la mencionada ausencia de una coalición mayoritaria estable-. Esta comisión recibió del relator un complejo sustitutivo con 264 artículos en las disposiciones permanentes y 72 en las transitorias. Se abre un nuevo proceso de presentación de enmiendas y de febril actividad constituyente del que resultan más de 10.000 enmiendas ${ }^{25}$. Después de un conflictivo proceso de sistematización, sobre cuyo contenido los "progresistas" habían hecho valer la artificial mayoría que disponían entre los 93 miembros de la comisión ${ }^{26}$, se avecinaba la hora de volver al pleno para las votaciones finales, quedando claro el empate y, por tanto, la necesidad de negociar, ya que

"como no tenías mayoría para votar nada, tenías que estar sobre el argumento. Fue frente a esto que surgieron las reuniones previas de los responsables de Grupo para comenzar a intentar organizar el proceso de votación porque, hasta entonces, no se habían reunido (...) surgió entonces la necesidad de que los responsables de los distintos grupos se sentaran en la mesa para concretar las materias consensuales en primer lugar e identificar las materias no consensuales. Surgió así la Junta de Portavoces que empezó a dominar el proceso" 27.

Esta Junta de Portavoces, estructurada de forma que posee inusuales poderes en una perspectiva comparada, no sólo no se extinguió después de la promulgación de la Constitución sino que además se convirtió en una de las principales instancias de coordinación del proceso legislativo. Sus atribuciones, informales en el periodo constituyente, recibieron la rúbrica del Reglamento Interno de la Cámara de Diputados, que incluso las amplió. El nuevo Reglamento Interno es producto de los nuevos poderes que la Constitución otorga al Poder Legislativo. Además de la Junta de Portavoces, la práctica constituyente legó una cierta parsimonia en la creación de Comisiones. En el periodo anterior a 1987, la Cámara contaba con 23 comisiones técnicas. Como la Constituyente había funcionado con ocho comisiones temáticas, en el periodo posterior fue posible disminuir el número de comisiones de 23 a 13 . A pesar del rechazo de una propuesta que estipulaba un máximo de ocho comisiones, que se estructurarían como sesiones miniplenarias temáticas ${ }^{28}$, la disminución adoptada mostró la disposición de contener el impulso desagregador presente en la estructura anterior, que varios parlamentarios atribuyen al

"deseo que tienen los diputados de multiplicar el $n$ mero de presidencias (...)[dado que] la figura de presidencia es atractiva. Hay mucha gente que quiere ser presidente de la comisión y esto provoca una proliferación muy dañina. $^{29}$

(24) Treinta mil electores podían suscribir una propuesta organizada sobre la responsabilidad de, por lo menos, tres entidades asociativas. Un relato detallado de este proceso puede ser encontrado en el trabajo de J.G. Lucas Coelho, "O Processo Constituiente", en Memória Fotográfica do Processo Constituiente, Ed.Agir, 1988, Brasilia.

(25) Según el estudio de la nota anterior, fueron presentadas 65.809 enmiendas en todo el proceso.

(26) Originalmente la comisión tenían 89 miembros, pero para asegurar la presencia de por lo menos un miembro de cada partido, independientemente del tamaño de su bancada, ella acabó reuniendo 93 parlamentarios.

(27) Diputado Nelson Jobim (PMDB-RS), en declaración en el CEBRAP en junio de 1993.

(28) Propuesta del diputado José Genoíno (PT-SP)

(29) Diputado Roberto Campos (PPR-RJ). 
Más allá de la presidencia,

“cuanto más comisiones mejor (...), porque (...) tienes un vicepresidente más, una secretaria más, una cuenta telefÛnica más ... ${ }^{30}$

Cambios como los de la reglamentación de las atribuciones de la Junta de Portavoces y la disminución del número de Comisiones no fueron suficientes para neutralizar la atomización del proceso legislativo. Con la elección de una nueva Mesa para los dos primeros años de la legislatura posterior (1991-1994), fue nombrada una comisión parlamentaria destinada a ofrecer a la Mesa estudios y sugerencias con el objetivo de perfeccionar los trabajos administrativos y legislativos de la Cámara, la llamada Comisión de Modernización. El fracaso de esta iniciativa, inspirada en el periodo constituyente, se debe, principalmente, a su intento de eliminar esta Junta de Portavoces, de crear una Comisión de Selección (que evaluaría preliminarmente la relevancia de los proyectos presentados) y prohibir la contratación de parientes de diputados para cargos de confianza en la Cámara.

\section{B. Las nuevas prerrogativas del legislativo}

La nueva Constitución fue la culminación de la lucha por el Estado de derecho en Brasil. Buena parte de los esfuerzos realizados se destinaron tanto a devolver al Legislativo las prerrogativas perdidas durante la dictadura, como a fortalecerlo en su capacidad de influir en los rumbos del país, que comparada con los recursos de poder que nuestra tradición siempre otorgó al Ejecutivo central, era ya pequeña incluso antes del periodo militar. Desde esta perspectiva, la gran novedad es el nuevo mecanismo de elaboración del Presupuesto General de la Unión, que pasa a ser compartido con el Legislativo por medio de la Comisión Mixta de Presupuesto, que reúne senadores y diputados. Sin espacio para detenernos en este asunto, nos limitamos a señalar que ésta tiene un considerable poder para alterar la propuesta presupuestaria elaborada por el Ejecutivo, dado que sus miembros tienen margen para reubicar las partidas destinadas a inversión dentro de los distintos ministerios. En este proceso se da una febril actividad de producción de enmiendas, que en 1992, por ejemplo, fueron más de 75 mil, cerca de 150 enmiendas por miembro. Este particular ilustra bien la magnitud del obstáculo que representa nuestra tradición parlamentaria para una recepción provechosa de las recientes innovaciones institucionales.

Otra importante innovación fue la institucionalización del poder concluyente de las comisiones técnicas, única materia del proceso legislativo que recibió tratamiento constitucional. La nueva prerrogativa permite que, en ciertas materias, las comisiones aprueben proyectos de ley sin que estos precisen enfrentar la lista de espera para su votación en Pleno, salvo que exista un recurso contrario apoyado por un mínimo de un décimo de los miembros de la Casa, en un plazo de cinco sesiones. La extinción del Decreto-ley, que permitía al Ejecutivo un amplio ejercicio de la capacidad de legislar, fue otra cambio importante. Fue sustituido por la figura de la Medida Provisoria, que mantiene la capacidad del ejecutivo para enfrentar situaciones de emergencia, pero con la exigencia de apreciación del Legis-

(30) Diputado José Genoíno (PT-SP), en declaración en CEBRAP en junio de 1993 lativo, dejando de estar éste sobre la amenaza de "curso de plazo", expediente del periodo militar que ante el no pronunciamiento del Legislativo en un plazo dado, aprobaba la iniciativa del Ejecutivo de forma automática. La figura del "Requerimiento de Información" otorgó una notable capacidad de seguimiento al legislativo sobre las acciones y asuntos del Ejecutivo, ya que cualquier diputado, teniendo su petición aprobada por el Pleno, puede exigir informaciones pormenorizadas al Poder Ejecutivo, bajo la pena para la autoridad requerida de incurrir en crimen de responsabilidad. Finalmente, merecen ser destacadas las proposiciones de Fiscalización y Control que, siendo presentadas por cualquier diputado a la comisión competente y siendo aprobada por ésta, permiten acompañar las actos del poder Ejecutivo ${ }^{31}$.

No se puede dejar de señalar que, a pesar de estas innovaciones, la Presidencia de la República mantiene un tremendo poder de iniciativa: ha sido responsable del 78,6\% de los proyectos que se convirtieron en ley a lo largo del periodo 1989-1992 — cabe indicar que el 23\% de éstos eran Medidas Provisorias, que pueden ser modificadas por el Legislativo, como es el caso de las peticiones de créditos (64\% de las iniciativas de la Presidencia)—. Sin embargo era nuestra intención iluminar en qué medida el potencial innovador contenido en estas nuevas disposiciones institucionales fue neutralizado por el individualismo y el centralismo del perfil bifronte de nuestra vida parlamentaria, y no tanto de analizar la indiscutible supremacía propositiva del Ejecutivo.

\section{Redistribución de Tributos y Transferencias}

La principal característica de la transformación tributaria establecida en la Constitución de 1988 es la descentralización de los recursos en favor de los estados y los municipios, éstos últimos en particular. La Constituyente no hizo más que consagrar una tendencia que venía emergiendo con la década del 80, en la senda de la redemocratización, especialmente con el restablecimiento, en 1982, del voto directo para gobernadores, derecho extendido posteriormente, en 1985, a la elección de los alcaldes de las capitales de estado y grandes ciudades.

Esa redistribución de recursos en favor de los niveles subfederales de gobierno tiene, respecto del papel desarrollado por los diputados, dos repercusiones políticas importantes. En primer lugar, el Ejecutivo central ya no puede responder de la misma forma y, sobre todo, con la misma magnitud, a las reivindicaciones de los diputados. Para que se tenga una idea de la dimensión de esta transformación, las transferencias negociadas de la Unión para los Estados, no vinculadas constitucionalmente, fueron estimadas, en 1991, en menos de la mitad del montante de 1988, lo que significa menos del $20 \%$ de las transferencias. En segundo lugar, los ejecutivos de las unidades subfederales aumentan su autonomía, ya que disponen de más recursos y pueden desarrollar nuevas pautas de relación con los diputados federales de sus estados. Cerca del $80 \%$ de las transferencias son constitucionales, no dependen de la negociación y, además, progresivamente vienen siendo liberadas de cualquier condicionalidad en la aplicación: cerca del $60 \%$ del total de esas transferencias se realizan bajo esta situación ${ }^{32}$.

(31) Este párrafo se apoya en el texto "O funcionamiento do Congresso Nacional", de Regina Balieiro Devescovi, CEBRAP, mimeo, marzo de 1993

(32) Afonso, José R.R., "Aspectos Conceituais das Relazoes Financieras Intergobernamentais", en Estudios Económicos, nJ1, S.P, 1992. 
Esta limitación de la discrecionalidad del Ejecutivo central retira un insumo importante para desarrollar la coordinación prebendataria que los responsables de los Grupos parlamentarios gubernamentales ejercían sobre sus compañeros ya que cada vez hay menos con lo que contentar atomizadamente a los diputados. Como relata un parlamentario que sigue el asunto,

"La constitución reduce la capacidad de inversión de la

Unión. (...) Cuando los parlamentarios comenzaron a sentir que no había más dinero para buscar en el Ejecutivo, estaban volviendo al pleno y alli formaban aquello que Ulisses (Guimaraes, ex-presidente de la Constituyente) llamaba el "valle de los caídos", que eran los diputados que se sentaban detrás de la $25^{a}$ fila, y que eran totalmente ajenos a lo que allí estaba aconteciendo. ${ }^{33 "}$

Este "retorno al Pleno" expresa la creciente insatisfacción de los diputados con la autonomía que disfruta la Junta de Portavoces, pues los métodos de la fase anterior ya no pueden mantenerse sin el apoyo de los recursos que antes intermediaban.

\section{CONCLUSIÓN}

Escribimos la Conclusión de este ensayo en el momento en que la sociedad brasileña asiste a los trabajos de la Comisión Parlamentaria de Investigación (CPI) que estudia la corrupción en la Comisión Mixta del Presupuesto. Este escándalo ilustra a la perfección el hibridismo de la Cámara, consistente en la permanencia anacrónica de prácticas pretéritas junto a la vigencia de nuevas reglas. Por eso es interesante analizarlo en retrospectiva para captar sus puntos de anclaje en viejas dinámicas. En la Introducción dijimos que en la vida interna de la Cámara entran en conflicto aspectos de la tradición heredada del periodo autoritario, innovaciones institucionales salidas de la Constituyente e influencias externas generadas por el ordenamiento legal que rige las elecciones y la formación de los partidos. A lo largo del texto exploramos como estas lógicas implican una estructuración institucional donde se mezclan fragmentación y agregación. Mediante la primera los diputados ejercen un irrestricto derecho de proponer, y por la segunda, apoyándose en la primera, los notables toman las decisiones, constituyendo un círculo de "delegación centralizadora". En consonancia con la democracia y con los nuevos dispositivos institucionales que rigen el Parlamento, hay tentativas de cambiar este estado de cosas, como por ejemplo la Comisión de Modernización, cuyo fracaso fue mencionado en el Final del apartado III y ahora puede ser retomado con todo su significado. En efecto, de las alteraciones propuestas por la Comisión, dos eran centrales y no fueron aceptadas: la extinción pura y simple de la Junta de Portavoces y la creación de una Comisión de Selección. Es curioso el hecho de que, mientras tanto, el Pleno haya rechazado ambas propuestas, que aparentemente señalan sentidos opuestos: la primera valoraba el papel del Pleno, quitando poder a los "notables"; la segunda recortaba el alcance de la acción individual, dando poder a la Comisión de Selección. Estas propuestas fueron derrotadas precisamente porque afectaban a los dos extremos del problema: a los "notables" les interesa el poder, no la racionalización del trabajo legislativo; a los diputados les interesa la irrestricta libertad de acción y de propuesta, no la toma de decisiones en sí. La

(33) Diputado Nelson Jobim (PMDB-RS). delegación de poder que la masa de diputados hace a los notables refleja la falta de compromiso con el proceso legislativo y no una disposición de prudencia en el empleo de las posibilidades individuales para condensarlo. Por ello, ambas fuerzas se "unieron" para derrotar los cambios y permanecen juntas, por ejemplo, en la Comisión Mixta de Presupuesto.

El Congreso puso en marcha la prerrogativa de enmendar el presupuesto sin que hubiesen sido revisadas las prácticas procedentes del circuito de intermediación con el Ejecutivo, en torno de la cual se articulaba la avidez atomizada de los diputados al servicio de la concentración de los notables. La innovación prevista en la constitución fue colonizada por la acción de estos intermediarios tradicionales, que convirtieron en privilegio una prerrogativa del Poder Legislativo. Dos entrevistas concedidas a esta investigación (muchos meses antes de que se pudiese imaginar la instalación de la CPI) ya apuntaban ese escenario en el que lo viejo no muere y lo nuevo no tiene fuerza para nacer. En la primera, cuando era interrogado a cerca de las razones de por qué fue derrotado en la elección para líder del PMDB, el diputado Odacyr Klein lamentaba

"la interferencia del Ejecutivo, vía Fiuza". Sin duda ninguna hubo interferencia de las grandes constructoras, porque algunos parlamentarios fueron buscados específicamente por éstas, parlamentarios que votaron conmigo y me lo contaron (...)

[Pregunta. ¿Por qué el interés de las constructoras en esto?]

-En función de la Comisión de Presupuesto, que aprobaba fondos y puede aprobar recursos específicos y determinados. Una de mis propuestas era alterar profundamente la Comisión de Presupuesto(...)

\section{[Pregunta: ¿en que dirección esa alteración?].}

-Primero, promover una rotación prácticamente total, para sacar de la Comisión al llamado grupo de los siete enanos $^{35}$

La segunda entrevista es la del diputado Paulo Bernardo, del PT, que lamentaba la cobertura realizada por los medios de comunicación respecto a la Comisión de Presupuesto, pues éstos perseguían solamente

"el asunto de las subvenciones sociales [aprobadas], sin tramitación formal y sin discusión. Cada parlamentario tiene derecho a una pequeña cantidad de recursos que destina para las Entidades Sociales de su región de origen, más allá de que haya sido él quien fundó la entidad. No es exacto, pero este año esto significó en torno a 700 millones de cruceiros, jen un presupuesto de 250 mil millones de dólares! No hace falta decir que es una pequeña gota para contentar al populacho mientras la cosa mucho mayor es aprobada sin discusión".

Estas declaraciones se complementan. Amparada en la tradición, la masa de diputados se despreocupa de ejercer la prerrogativa conquistada, alienándola a cambio de la porción clientelística. A los que conceden tales privilegios se delega el pésimo manejo de la "cosa mucho mayor", objeto de deseo de las empresas constructoras. Así, mientras las altas esferas diseñan el presupuesto, la masa de diputados ejerce toda su presumida

(34) El diputado Ricardo Fiuza, ex-lider del PFL, ex-ministro del Gobierno Collor, ex-relator del Presupuesto, es acusado hoy por la CPI de relaciones espurias con las grandes empresas constructoras.

(35) Denominación dada por la prensa a los siete miembros de esta comisión, todos de baja estatura, que se turnaban. rotando en los puestos clave de la Comisión, evitando dejarlos. El diputado Genebaldo Correa (PMDB-BA), es uno de estos diputados investigados, y pesan sobre el evidencias tremendas. 
independencia haciendo enmiendas al detalle de las subvenciones y condenando el curso legislativo a toda clase de irrelevancias. Las fuerzas que se enfrentan a este estado de cosas vienen creciendo a medida que, con el paso del tiempo, ellas se adueñan de los instrumentos institucionales ofrecidos por las nuevas reglas, cuyo ejercicio pone al desnudo el anacronismo de aquellas prácticas y la necesidad de un nuevo juego. Para vencer, la nueva organización de los trabajos legislativos deberá atacar las dos puntas del problema: de un lado, redefinir el derecho individual de iniciativa, de tal manera que disminuyan los costos de la tramitación y estimular la producción colegiada, y de otro, y estrechamente relacionado con el esfuerzo anterior, superar la delegación centralizadora por una coordinación participativa, combinando eficiencia con democracia.
Los últimos acontecimientos de la lucha entre lo viejo y lo nuevo en el Congreso dejan cada vez más claro que el espectáculo exhibido por la CPI del Presupuesto no es un escándalo más. Al lado del Impeachment, este episodio evidencia el fracaso de un modelo de representación fundado en la delegación irrestricta ${ }^{36}$, desprovista de mediaciones democráticamente constituidas. Con el impeachment tuvimos la posibilidad de dejar atrás la mítica imagen del líder solitario que todo lo resuelve, figura que tuvo en Collor su zenit. Con la CPI tenemos la posibilidad de construir un nuevo Congreso. La ruptura tan cabal y simultánea de este anillo de los nibelungos se explica por la prolongada crisis de hegemonía que vive la sociedad brasileña. La definición democrática de una nueva hegemonía permitirá la edificación de otro andamiaje de mediaciones, que el tiempo llenará de nuevas costumbres y, entonces, de nuevas deformaciones.

\section{RESUMEN}

Determinados rasgos históricos de las instituciones políticas brasileñas -forma de gobierno y sistema electoral principalmente-inciden en la dinámica interna del Congreso. El autor analiza como se desarrollan las diversas actividades vinculadas a la producción legislativa y pone en evidencia una dinámica individualista que anula la posibilidad de desarrollar estrategias cooperativas. No obstante, el estudio de las votaciones sobre determinadas cuestiones niega la hipótesis de que esta dinámica elimine la existencia de un posicionamiento ideológico dentro del Congreso sobre el eje izquierda-derecha.

\section{ABSTRACT}

It is analyzed how specific historical features of brazilean political institutions -specially, type of government and electoral system- influence in the internal dynamic of Congress. The author analyzes the process of legislative production and underlines an individualistic dynamic which cancel the possibility to develop cooperative strategies. However, the study possibility to develop cooperative strategies. However, the study of votings about specific questions denyes the hypothesis which sets up that this kind of dynamic eliminates the existence of an ideological position inside the Congress in the axis left-right. 